Preprint DFPD 95/TH/37

hep-th/9506109

June, 1995

\title{
Duality symmetric actions with manifest space-time symmetriest
}

\author{
Paolo Pasti, Dmitrij Sorokin[ and Mario Tonin \\ Università Degli Studi Di Padova, Dipartimento Di Fisica "Galileo Galilei" \\ ed INFN, Sezione Di Padova, Via F. Marzolo, 8, 35131 Padova, Italia
}

\begin{abstract}
We consider a space-time invariant duality symmetric action for a free Maxwell field and an $S L(2, \mathbf{R}) \times S O(6,22)$ invariant effective action describing a low-energy bosonic sector of the heterotic string compactified on a six-dimensional torus. The manifest Lorentz and general coordinate invariant formulation of the models is achieved by coupling dual gauge fields to an auxiliary vector field from an axionic sector of the theory.

PACS numbers: 11.15-q, 11.17+y
\end{abstract}

\footnotetext{
${ }^{1}$ This work was carried out as part of the European Community Programme "Gauge Theories, Applied Supersymmetry and Quantum Gravity" under contract SC1-CT92-D789, and supported in part by M.P.I.

${ }^{2}$ on leave from Kharkov Institute of Physics and Technology, Kharkov, 310108, Ukraine.

e-mail: sorokin@pd.infn.it

${ }^{3} \mathrm{e}-$ mail: tonin@pd.infn.it
} 


\section{Introduction}

The understanding of the important role played by duality in (super)-Yang-Mills theories 11, 2, 3], supergravity and the theory of strings [4- [7, 8] has allowed one to make new insight into the structure of these theories and to find deep relationship between their different (dual) versions. If target space duality (T-duality) and S-duality (which is the generalization of the electric-magnetic duality) are the exact symmetries of string theory [4, 5, 6], it is natural to assume that there should be a version of the theory where these duality symmetries are manifest.

A T-duality symmetric string action was proposed by Tseytlin [9] and generalized to a case of the heterotic string by Schwarz and Sen [10]. This required modification of spacetime transformations of fields. Note, however, that effective supersymmetric field actions which describe the low-energy behavior of superstrings do have a global non-compact symmetry related to $\mathrm{T}$-duality, while $\mathrm{S}$-duality is only a symmetry of the equations of motion [1]-13.

In 13 Schwarz and Sen proposed models for describing antisymmetric gauge fields in D-dimensional space-time, where S-duality symmetry was lifted to the level of action, their results being the generalization of earlier work by Floreanini and Jackiw [14] and Henneaux and Teitelboim [15] who constructed actions for describing self-dual tensor fields in $(4 \mathrm{p}+2)$-dimensional space-time $(\mathrm{p}=0,1, .$.$) . The general feature of the models$ [9, 10, 13, 14, 15] is that, due to the explicit fixing of the time direction, they loose manifest Lorentz and general coordinate invariance, which, however, are replaced by some modified transformations. This is an example how duality effects the symmetry structure of the theory.

Anyway, one may try to look for a formulation of duality symmetric actions in which conventional space-time symmetries are restored. An attempt to do this for a duality symmetric version of Maxwell theory [13] t] was undertaken in [17]. Having been inspired by this paper we proposed in [18] a manifestly space-time and duality symmetric formulation of (supersymmetric) Maxwell theory by enlarging the Schwarz-Sen model [13] with an auxiliary vector field $u_{m}(x)$ and an antisymmetric tensor field $\Lambda_{m n}(x)(\mathrm{m}=0,1,2,3)$ in such a way that upon solving for equations of motion and gauge fixing additional local symmetries associated with the auxiliary fields the model [18] could be reduced to that of Schwarz and Sen. The physical nature of $u_{m}(x)$ and $\Lambda_{m n}(x)$ was supposed to be a relic of a gravitational vielbein and an axion-like potential, respectively.

In the present paper we further develop the duality symmetric model of [18. In particular, we will show that the model indeed has a local symmetry (anticipated in [18]) allowing one to choose $u_{m}(x)$ to be a constant unit-norm time-like vector and, thus, to demonstrate that the duality symmetric models [13], with their non-conventional spacetime symmetries, correspond to a definite gauge choice for auxiliary fields in corresponding duality symmetric models with ordinary Lorentz and general coordinate invariance. Using the example of a model for two abelian gauge fields in $\mathrm{D}=1+3$, we shall show that duality between these two fields arises due to their specific coupling to a pseudoscalar sector of the theory through the field $u_{m}(x)$, and the latter can be gauge fixed to a constant timelike vector by use of a local counterpart of a Peccei-Quinn symmetry of axion models. Thus, $u_{m}(x)$ can originate, in fact, from the pseudoscalar sector of the theory and, at the same time, carry some properties of a local Lorentz frame field. In Section 3 we will

\footnotetext{
${ }^{4}$ Earlier an alternative version of duality invariant Maxwell action was considered in 16]
} 
present a space-time and $S L(2, \mathbf{R}) \times O(6,22)$ invariant effective action which describes a low-energy limit of a toroidally compactified heterotic string.

\section{Duality symmetric Maxwell action}

Let us start with an action describing a free Maxwell field $A_{m}(x)$ and a pseudoscalar ('axion') field $a(x)$ in $\mathrm{D}=1+3$ Minkowski space:

$$
S=\int d^{4} x\left(-\frac{1}{4} F_{m n} F^{m n}-\frac{1}{2}\left(\partial_{m} a(x)-u_{m}(x)\right)\left(\partial^{m} a(x)-u^{m}(x)\right)-\epsilon^{p q m n} u_{p} \partial_{q} \Lambda_{m n}\right)
$$

where the first term is the ordinary Maxwell Lagrangian with $F_{m n}=\partial_{m} A_{n}(x)-\partial_{n} A_{m}(x)$ and the last two terms form the Lagrangian which, upon solving the equations of motion for $u_{m}(x)$ and using a local symmetry of (1) under transformations

$$
\delta a(x)=\varphi(x), \quad \delta u_{m}(x)=\partial_{m} \varphi(x),
$$

produces (see, for the details $19,20,21,22,23$ and references therein) the free Lagrangian for the scalar field $a(x)$

$$
L=-\frac{1}{2} \partial_{m} a(x) \partial^{m} a(x)
$$

or its dual

$$
L=\frac{1}{3 !} \partial_{[m} \Lambda_{n p]} \partial^{[m} \Lambda^{n p]},
$$

with the duality relation between $a(x)$ and the antisymmetric field $\Lambda_{n p}$ to be

$$
\partial^{l} a(x)=\epsilon^{l m n p} \partial_{m} \Lambda_{n p}
$$

The role of $u_{m}(x)$ in (11) is to be the gauge field of the symmetry (2) and to ensure (5) on the mass shell.

Besides (国) the action (四) is invariant under the abelian gauge transformations of $A_{m}(x)$ and $\Lambda_{m n}(x)$ :

$$
\delta A_{m}(x)=\partial_{m} b(x), \quad \delta \Lambda_{m n}=\partial_{[m} b_{n]}(x) .
$$

Note that without violating local symmetry (2) one can couple fields from the pseudoscalar sector of (1) to the gauge field in an axion-like fashion:

$$
S_{\text {int }}=-\int d^{4} x\left(\partial_{m} a-u_{m}\right) \epsilon^{m n l p} A_{n} F_{l p}
$$

The sum of (11) and (17) is explicitly invariant under (2). It is also invariant under the gauge transformations (6) if one requires that the variation of $\Lambda_{m n}$ acquires the contribution $\delta_{b} \Lambda_{m n}=b(x) F_{m n}$. Eliminating either $u_{m}$ and $\Lambda_{m n}$ or $u_{m}$ and $a(x)$ by use of equations of motions and local symmetries one gets the dual versions of the axion theory [20, 21, 22, 23].

Now the question arises whether it is possible to replace (1) with an action which would be duality symmetric in the Maxwell field sector, still possess ordinary Lorentz invariance and be equivalent (at least classically) to the action (1). The answer turns out to be positive [18]. 
From [13\| we learn that for making the electric-magnetic duality manifest at the level of action one has to double the number of abelian fields (introducing $A_{m}^{\alpha}(x)(\alpha=1,2)$ ) and construct a duality symmetric action in such a way that equations of motion obtained from this action lead to the vanishing of the self-dual tensor

$$
\mathcal{F}_{m n}^{\alpha}=\mathcal{L}^{\alpha \beta} F_{m n}^{\beta}-F_{m n}^{* \alpha}=\frac{1}{2} \epsilon_{m n p q} \mathcal{L}^{\alpha \beta} \mathcal{F}^{\beta p q}
$$

where $\mathcal{L}^{\alpha \beta}=-\mathcal{L}^{\beta \alpha}\left(\mathcal{L}^{12}=1\right), F_{m n}^{* \alpha}=\frac{1}{2} \epsilon_{m n l p} F^{l p \alpha}$.

When

$$
\mathcal{F}_{m n}^{\alpha}=0
$$

one of the abelian fields becomes completely determined through another one, and for the latter we get the free Maxwell equations by differentiating (9). Then the duality (9) between the two gauge fields reduces to the duality between the electric and magnetic strength of the gauge field which has been chosen to be independent.

The duality symmetric action proposed in [13], which gives (9), has the following form:

$$
S=-\frac{1}{2} \int d^{4} x\left(B^{i \alpha} \mathcal{L}^{\alpha \beta} E_{i}^{\beta}+B^{i \alpha} B_{i}^{\alpha}\right)
$$

where

$$
E_{i}^{\alpha}=F_{0 i}^{\alpha}=\partial_{0} A_{i}^{\alpha}-\partial_{i} A_{0}^{\alpha}, \quad B^{i \alpha}=\frac{1}{2} \varepsilon^{i j k} F_{j k}^{\alpha}=\varepsilon^{i j k} \partial_{j} A_{k}^{\alpha},
$$

and $i, j, k=1,2,3$ are spacial indices. The action (10) is invariant under modified spacetime transformations of $A_{i}^{\alpha}$ (in the gauge $A_{0}^{\alpha}=0$ ):

$$
\delta A_{i}^{\alpha}=x^{0} v^{k} \partial_{k} A_{i}^{\alpha}+v^{k} x^{k} \partial_{0} A_{i}^{\alpha}+v^{k} x^{k} \mathcal{L}^{\alpha \beta} \mathcal{F}_{0 i}^{\beta},
$$

where the first two terms describe the ordinary Lorentz boosts along a constant velocity $v^{i}$ and the third term vanishes on the mass shell (9).

The model constructed this way [13 is classically and quantum mechanically 24 equivalent to the free Maxwell theory.

The covariantization of (10) is achieved by coupling the self-dual tensor (8) to the auxiliary field $u_{m}(x)$ from the pseudoscalar part of the Lagrangian (11) as follows:

$$
\begin{aligned}
S & =\int d^{4} x\left(-\frac{1}{8} F_{m n}^{\alpha} F^{\alpha m n}+\frac{1}{4\left(-u_{l} u^{l}\right)} u^{m} \mathcal{F}_{m n}^{\alpha} \mathcal{F}^{\alpha n p} u_{p}\right. \\
& \left.-\frac{1}{2}\left(\partial_{m} a-u_{m}\right)\left(\partial^{m} a-u^{m}\right)-\epsilon^{m n p q} u_{m} \partial_{n} \Lambda_{p q}\right) .
\end{aligned}
$$

Action (13) differs from that considered in [18] at the following point. In 18 $u_{m}(x)$ was required to have the negative unit norm

$$
u^{2} \equiv u_{m} u^{m}=-u_{0} u_{0}+u_{i} u_{i}=-1
$$

and played the role of a component of a local Lorentz frame. In (13) we weakened the normalization condition by introducing the norm of $u_{m}(x)$ only into the term containing $\mathcal{F}_{m n}^{\alpha}$. We shall demonstrate the relationship between the two versions of the model later on. 
The necessity to introduce the norm of $u_{m}(x)$ into the $\mathcal{F}_{m n}^{\alpha}$-term is dictated by the requirement to preserve the local symmetry (2). The action (13) is invariant under the transformations (2) provided $A_{m}^{\alpha}(x)$ and $\Lambda_{m n}(x)$ are transformed as follows

$$
\delta A_{m}^{\alpha}=\frac{\varphi(x)}{u^{2}} \mathcal{L}^{\alpha \beta} \mathcal{F}_{m n}^{\beta} u^{n}, \quad \delta \Lambda_{m n}=\frac{\varphi(x)}{\left(u^{2}\right)^{2}} \mathcal{F}_{m}^{\alpha r} u_{r} \mathcal{F}_{n}^{\beta s} u_{s} \mathcal{L}^{\alpha \beta}
$$

Then, since the solution to the equation of motion of $\Lambda_{m n}$, obtained from (13), is

$$
u_{m}(x)=\partial_{m} \hat{\varphi}(x),
$$

where $\hat{\varphi}(x)$ is a scalar function, we can use the transformations (2) to put $u_{m}=\delta_{m}^{0}$. I In this gauge the action (13) reduces to (10), and the transformation of $A_{m}^{\alpha}$ in (15) (with $\left.\varphi=x^{i} v^{i}\right)$ is combined with the corresponding Lorentz transformation producing (12).

We see that $u_{m}(x)$ plays a double role. From the one hand side it is the gauge field of local Peccei-Quinn symmetry and from the other hand it corresponds to a component of a local Lorentz frame.

The action (13) has another local symmetry [18] (which generalizes that of (10) [13]) under the following transformations of $A_{m}^{\alpha}$ and $\Lambda_{m n}$ :

$$
\begin{gathered}
A_{m}^{\alpha} \rightarrow A_{m}^{\alpha}+u_{m} \varphi^{\alpha}(x) \\
\Lambda_{m n} \rightarrow \Lambda_{m n}-\frac{\varphi^{\alpha}}{u^{2}} \mathcal{F}_{m p}^{\alpha} u^{p} u_{n}+\frac{\varphi^{\alpha}}{u^{2}} \mathcal{F}_{n p}^{\alpha} u^{p} u_{m}
\end{gathered}
$$

This symmetry allows one to reduce the general solution of the equations of motion of $A_{m}^{\alpha}$

$$
\epsilon^{l m n p} \partial_{m}\left(u_{n} \mathcal{F}_{p r}^{\alpha} u^{r}\right)=0
$$

to eq. (9) (see [13, 18 for the details). In the gauge where $\mathcal{F}_{m n}^{\alpha}=0$, the equations of motion of $u_{m}$ lead to the same expressions for $a(x)$ and $\Lambda_{m n}$ that follow from (11).

To transit from (13) to (1) we must solve eqs. (18) for one of the gauge fields in terms of another one and substitute the solution back to (13) [13, 18]. If we skip the kinetic term for $a(x)$ in (11) and (13), $\Lambda_{m n}$ becomes a pure gauge as well, and the three actions (11), (10) and (13) become classically equivalent and describe dynamics of a single Maxwell field.

Now we shall demonstrate how the action (13) is related to the version considered in [18]. There the vector field $u_{m}$ (in (13)) was subjected to the normalization condition (14) (we shall denote the normalized vector as $\hat{u}_{m}$ ). This caused a problem of establishing the explicit invariance of the model under the transformations (2), (15). To ensure this invariance one should couple (13) with the normalized $\hat{u}_{m}$ 18 to scale invariant gravity 25]. Then the action takes the form (we skip, for simplicity, the kinetic term of $a(x)$ ):

$$
\begin{gathered}
S=\int d^{4} x \sqrt{-g}\left(-\frac{1}{8} F_{m n}^{\alpha} F^{\alpha m n}+\frac{1}{4} \hat{u}^{m} \mathcal{F}_{m n}^{\alpha} \mathcal{F}^{\alpha n p} \hat{u}_{p}\right. \\
\left.-\frac{1}{\sqrt{-g}} \epsilon^{p q m n} \hat{u}_{p} \partial_{q} \Lambda_{m n}+R \Phi^{2}+6 \partial_{m} \Phi \partial^{m} \Phi\right),
\end{gathered}
$$

\footnotetext{
${ }^{5}$ To escape singularities we should require the norm of $u_{m}$ to be nonzero.
} 
where $g_{m n}(x)$ is a metric, $g=\operatorname{det} g_{m n}, R(x)$ is the scalar curvature and $\Phi(x)$ is a conformal scalar field. The action (19) is invariant under (2), (15) provided $g_{m n}(x)$ and $\Phi(x)$ subject to the following scale transformations:

$$
\delta g_{m n}(x)=\left(\hat{u}^{m} \partial_{m} \varphi\right)^{2} g_{m n}(x), \quad \delta \Phi(x)=-\left(\hat{u}^{m} \partial_{m} \varphi\right) \Phi(x) .
$$

Making redefinition of the metric and $\hat{u}_{m}$ as follows

$$
g^{m n}(x) \rightarrow \Phi^{2} g^{m n}, \quad \hat{u}_{m}(x) \rightarrow \frac{1}{\Phi} \hat{u}_{m}
$$

(which preserves the condition (14) 5) we can rewrite (20) in the form which describes coupling the model to the Einstein gravity:

$$
S=\int d^{4} x \sqrt{g}\left(-\frac{1}{8} F_{m n}^{\alpha} F^{\alpha m n}+\frac{1}{4} \hat{u}^{m} \mathcal{F}_{m n}^{\alpha} \mathcal{F}^{\alpha n p} \hat{u}_{p}-\frac{1}{\sqrt{g}} \Phi \epsilon^{p q m n} \hat{u}_{p} \partial_{q} \Lambda_{m n}+R\right) .
$$

The only place where $\Phi(x)$ is present in (22) is the term $\Phi \epsilon^{p q m n} \hat{u}_{p} \partial_{q} \Lambda_{m n}$. Then, putting $\Phi(x) \hat{u}_{m}(x)=u_{m}(x)$, making use of (14) and taking the flat limit we get the action (13).

This concludes the establishment of the links between different versions of the duality symmetric formulation of free Maxwell theory.

\section{Low-energy effective action in string theory with manifest $S L(2, \mathbf{R}) \times O(6,22)$ and space-time symme- try}

In this section we present manifest space-time invariant generalization of the $S L(2, \mathbf{R}) \times$ $O(6,22)$ invariant low-energy effective action [13] describing heterotic string theory compactified on a six-dimensional torus [12, 26]. To do this we should introduce 28 dual pairs 27] $A_{m}^{\alpha, a}(a=1, \ldots, 28)$ of abelian gauge fields and couple them to scalar fields in an $S L(2, \mathbf{R}) \times O(6,22)$ covariant way. This is achieved by modifying the self-dual tensor (8) as follows:

$\mathcal{F}_{m n}^{\alpha, a}=\mathcal{L}^{\alpha \beta} L^{a b} F_{m n}^{\beta, b}-\left(\mathcal{L}^{T} \mathcal{M} \mathcal{L}\right)^{\alpha \beta}\left(L^{T} M L\right)^{a b} F_{m n}^{* \beta, b} \equiv \frac{\sqrt{-g}}{2}\left(-\mathcal{L}^{T} \mathcal{M}\right)^{\alpha \beta}\left(L^{T} M\right)^{a b} \epsilon_{m n p q} \mathcal{F}^{\beta, b} p q$

where $2 \times 2$ matrix valued scalar field

$$
\mathcal{M}=\frac{1}{\lambda_{2}(x)}\left(\begin{array}{cc}
1 & \lambda_{1}(x) \\
\lambda_{1}(x) & \lambda_{1}^{2}+\lambda_{2}^{2}
\end{array}\right)
$$

satisfies the following conditions:

$$
\mathcal{M}^{T}=\mathcal{M}, \quad \mathcal{M L} \mathcal{M}^{T}=\mathcal{L} .
$$

$\mathcal{M}, \mathcal{L}$ and $A_{m}^{\alpha, a}$ transform under the global $S L(2, \mathbf{R})$ transformations $\omega$ as follows:

$$
\mathcal{M} \rightarrow \omega^{T} \mathcal{M} \omega \quad \omega \mathcal{L} \omega^{T}=\mathcal{L} \quad A_{m}=\omega^{T} A_{m}
$$

\footnotetext{
${ }^{6}$ Note that one can introduce (14) into (19) with a Lagrange multiplier which transforms under the scale transformations in an appropriate way
} 
The $28 \times 28$ matrix-valued scalar field $M(x)$ satisfies the conditions

$$
M^{T}=M, \quad M^{T} L M=L,
$$

where

$$
L=\left(\begin{array}{ccc}
0 & I_{6} & 0 \\
I_{6} & 0 & 0 \\
0 & 0 & -I_{16}
\end{array}\right) .
$$

Under a global $O(6,22)$ rotation $M, L$ and $A_{m}^{\alpha, a}$ transform as follows:

$$
M \quad \rightarrow \quad \Omega^{T} M \Omega, \quad \Omega^{T} L \Omega=L \quad A_{m} \rightarrow \Omega^{T} A_{m} .
$$

(see ref. 13] for the details). The transformation law of the self-dual tensor (23) under $S L(2, \mathbf{R}) \times O(6,22)$ is

$$
\mathcal{F}_{m n} \rightarrow \omega^{-1} \Omega^{-1} \mathcal{F}_{m n}
$$

Using the properties of the fields described above one may convince oneself that the following general coordinate invariant action has $S L(2, \mathbf{R}) \times O(6,22)$ symmetry:

$$
\begin{aligned}
S & =\int d^{4} x \sqrt{-g}\left(-\frac{1}{8} F_{m n}^{\alpha, a}\left(\mathcal{L}^{T} \mathcal{M L}\right)^{\alpha \beta}\left(L^{T} M L\right)^{a b} F^{\beta, b m n}+\frac{1}{4 u^{2}} u^{m} \mathcal{F}_{m n}^{\alpha, a} \mathcal{M}^{\alpha \beta} M^{a b} \mathcal{F}^{\beta, b}{ }^{n p} u_{p}\right. \\
& \left.-\frac{1}{4} g^{m n} \operatorname{tr}\left(\partial_{m} \mathcal{M} \mathcal{L} \partial_{n} \mathcal{M} \mathcal{L}\right)+\frac{1}{8} g^{m n} \operatorname{Tr}\left(\partial_{m} M L \partial_{n} M L\right)-\frac{1}{\sqrt{g}} \epsilon^{p q m n} u_{p} \partial_{q} \Lambda_{m n}+R\right) .
\end{aligned}
$$

It can be rewritten in a simpler form

$$
\begin{aligned}
& S=\int d^{4} x \sqrt{-g}\left(\frac{1}{2 u^{2}} u^{m} F_{m n}^{* \alpha, a} \mathcal{F}^{\alpha, a n p} u_{p}-\frac{1}{\sqrt{-g}} \epsilon^{p q m n} u_{p} \partial_{q} \Lambda_{m n}+R\right. \\
& \left.-\frac{1}{4} g^{m n} \operatorname{tr}\left(\partial_{m} \mathcal{M L} \partial_{n} \mathcal{M L}\right)+\frac{1}{8} g^{m n} \operatorname{Tr}\left(\partial_{m} M L \partial_{n} M L\right)\right)
\end{aligned}
$$

which, upon fixing the gauge $u_{m}=\frac{1}{\sqrt{-g^{00}}} \delta_{m}^{0}, \Lambda_{m n}=0$, directly reduces to the SchwarzSen action [13].

Note that we did not add the kinetic term of $a(x)$ (11), (13) to the actions (31) and (32). This is because we would like to identify $a(x)$ with $\lambda_{1}(x)$ which has already entered the actions (31) and (32) as part of the dilaton-axion matrix $\mathcal{M}$ (24). The coupling of $\mathcal{M}$ to the gauge fields ensures the manifest $S L(2, \mathbf{R})$ symmetry but brakes local transformations of $\lambda_{1}(x)$ (or $a(x)$ in (2)) down to the global Peccei-Quinn shifts which form a subgroup of the global $S L(2, \mathbf{R})(26)$ [. Such a coupling violates duality between the pseudoscalar field and $\Lambda_{m n}$ in favor of the former and makes $\Lambda_{m n}$ an auxiliary field, which can be eliminated from (31), (32) by solving for the equations of motion of $u_{m}$ and $\Lambda_{m n}$, and substituting $u_{m}(x)$ back into (31), (32) in the form $u_{m}=\partial_{m} \hat{\varphi}(x)$ (16).

It is tempting to look for a version of the low-energy effective string action which would be manifestly duality symmetric not only in the gauge sector but in the axion sector (1) , (7) as well. Might it imply a localization of the $S L(2, \mathbf{R})$ ?

\footnotetext{
${ }^{7}$ Note that the action (31), (32) is still invariant under the local transformations of $u_{m}$ (2), $A_{m}$ and $\Lambda_{m n}$ (15)
} 


\section{Conclusion and discussion}

We have constructed the space-time invariant duality symmetric action for the free Maxwell theory and the $S L(2, \mathbf{R}) \times O(6,22)$ invariant effective action describing lowenergy bosonic sector of the heterotic string compactified on a 6 -dimensional torus. This has been achieved by coupling the self-dual stress tensor, constructed out of the dual gauge fields, to the auxiliary vector field from the axionic sector of the theory.

One can add to the bosonic action (13) the kinetic term for neutral fermions:

$$
S_{F}=-i \int d^{4} x \bar{\psi} \gamma^{m} \partial_{m} \psi
$$

Then the full action becomes supersymmetric [13, 18] under the following transformations with odd constant parameters $\epsilon^{\alpha}=i \gamma_{5} \mathcal{L}^{\alpha \beta} \epsilon^{\beta}$ :

$$
\begin{gathered}
\delta A_{m}^{\alpha}=i \bar{\psi} \gamma_{m} \epsilon^{\alpha}, \\
\delta \psi=\frac{1}{8} F^{\alpha m n} \gamma_{m} \gamma_{n} \epsilon^{\alpha}-\frac{1}{4 u^{2}} \mathcal{L}^{\alpha \beta} u_{p} \mathcal{F}^{\alpha p m} u^{n} \gamma_{m} \gamma_{n} \epsilon^{\beta},
\end{gathered}
$$

all other fields being inert under the supersymmetry transformations.

We see that the supersymmetric transformation law for the fermion $\psi(x)$ (34) is nonconventional and reduces to the ordinary one only on the mass shell (9). This reminds the problem with the Lorentz transformations (12) which we have just solved. Using the same reasoning as lead us to introducing $u_{m}(x)$ one may try to find a superpartner of $u_{m}(x)$ whose presence in the theory gives rise to a local fermionic symmetry (being a counterpart of (2, 15)) which involves $\psi(x)$ and leads to (34) upon gauge fixing the local fermionic symmetry. This construction may arise from coupling the models discussed above to supergravity, from which, in fact they originate.

The covariantization procedure for duality symmetric actions [14, 15, 9, 13] proposed in 18] and developed herein is applicable to abelian tensor fields in space-time dimensions other than $D=4$ and may turn out to be useful for finding new dual versions of $D=10$ supergravity [13.

Acknowledgements. The authors are grateful to I. Bandos for constant interest to this work and helpful comments. D.S. would also like to thank A. Khoudeir, F. Quevedo and A. Sen for useful discussion.

\section{References}

[1] C. Mantonen and D. Olive, Phys. Lett. B72, 117 (1977); P. Goddard, J. Nuyts and D. Olive, Nucl. Phys. B 125, 1 (1977); H. Osborne, Phys. Lett. B83, 321 (1979).

[2] M. Halpern, Phys. Lett. B81, 245 (1979); Phys. Rev. D19, 517 (1979).

[3] N. Seiberg and E. Witten, Nucl. Phys. B426, 19 (1994); Nucl. Phys. B431, 484 (1994);

N. Seiberg, The power of duality - exact results in 4D SUSY field theory. Preprint RU-95-37, IASSNS-HEP-95/46, hep-th/9506077 (and references therein). 
[4] K. Narain, Phys. Lett. B169, 41 (1986); K. Narain, H. Sarmadi and E. Witten, Nucl. Phys. B279, 369 (1987).

[5] A. Font, L. Ibañez, D. Lust and F. Quevedo, Phys. Lett. B249, 35 (1990); S. J. Rey, Phys. Rev. D43, 526 (1991).

[6] A. Shapere, S. Trivedi and F. Wilczek, Mod. Phys. Lett. A6, 2677 (1991).

[7] A. Sen, Nucl. Phys. B404, 109 (1993).

[8] J. H. Schwarz, String theory symmetries. Preprint CALT-68-1984, hep-th/9503127 (and references therein).

[9] A. Tseytlin, Phys. Lett. B242, 163 (1990); Nucl. Phys. B350, 395 (1991).

[10] J. H. Schwarz and A. Sen, Phys. Lett. B312, 105 (1993).

[11] M. de Roo, Nucl. Phys. B255, 515 (1985).

[12] J. Maharana and J. H. Schwarz, Nucl. Phys. B390, 3 (1993)

[13] J. H. Schwarz and A. Sen, Nucl. Phys. B411, 35 (1994).

[14] R. Floreanini and R. Jackiw, Phys. Rev. Lett. 59, 1873 (1987).

[15] M. Henneaux and C. Teitelboim, in Proc. Quantum mechanics of of fundamental systems 2, Santiago, 1987, p. 79; Phys. Lett. B206, 650 (1988).

[16] S. Deser and C. Teitelboim, Phys. Rev. D13, 1592 (1976); S. Deser, J. Phys. A: Math. Gen. 15, 1053 (1982).

[17] A. Khoudeir and N. Pantoja, hep-th/9411235.

[18] P. Pasti, D. Sorokin and M. Tonin, Phys. Lett. B352, 59 (1995); hep-th/9503182.

[19] V. I. Ogievetsky and I. V. Polubarinov, Soviet. J. Nucl. Phys. 4, 156 (1967); M. Kalb and P. Ramond, Phys. Rev. D9 2273 (1974).

[20] M. J. Bowick, S. B. Giddings, J. A. Harvey, G. T. Horowitz and A. Strominger, Phys. Rev. Lett. 61, 2823 (1988); S Coleman, J. Preskill and F. Wilczek, Nucl. Phys. B378, 175 (1992).

[21] H. Nicolai and P. K. Townsend, Phys. Lett. B98, 257 (1981).

[22] R. Kallosh, A. Linde, D. Linde and L. Susskind, Preprint SU-ITP-95-2, hep th/9502069.

[23] F. Quevedo, Preprint NEIP95-007, hep-th/9506081.

[24] I. Martin and A. Restuccia, Phys. Lett. B323, 311 (1994).

[25] S. Deser, Ann. Phys. 59, 248 (1970).

[26] A. Sen, Phys. Lett. B303, 22 (1993).

[27] R. Kallosh and T. Ortin, Phys. Rev. D48, 742 (1993). 